\title{
Treatment Options in Refractory West Syndrome
}

\author{
Prashant Jauhari $^{1}$ (D) Gautam Kamila $^{1}$
}

Received: 19 November 2019 / Accepted: 20 November 2019 / Published online: 6 December 2019

(C) Dr. K C Chaudhuri Foundation 2019

No other epilepsy syndrome better exemplifies intractability and recrudescence than West syndrome. Despite being known for more than $150 \mathrm{y}$, not much progress has occurred in the understanding of neural circuitry and epileptogenic mechanism of West syndrome. The resultant effect is a lack of an effective treatment plan targeting prolonged remission and preserved cognition. The only consensus that exists is that either hormonal therapy with ACTH or oral steroids or vigabatrin is to be used as first line agents [1]. The management protocol for cases not responding or relapsing on first line therapy is unclear. A host of literature is available regarding the various therapeutic options for refractory epileptic spasms. However, methodological inconsistencies, retrospective nature, small sample size and variability in the outcome measure studied have negatively impacted the development of a robust evidence base. Moreover, a significant proportion of reported cases from developed world have a non-structural etiology making it difficult to extrapolate the results to our population where hypoxic ischemic brain injury is a major contributor [2]. The potentially useful agents used in resistant West syndrome include valproate and benzodiazepine, topiramate, zonisamide, levetiracetam, ketogenic diet and surgery [3].

Nadig PL and colleagues have tried to prospectively find evidence of topiramate effectiveness in hormone/vigabatrin non-responsive West syndrome ( $87 \%$ with structural etiology). They found that $09 / 38$ had complete cessation of spasms but seven of these cases relapsed, bringing down the absolute effectiveness to $5 \%$. The authors concluded that topiramate had poor effectiveness in West syndrome and partly attributed this to long treatment lag and structural etiology [4].

Nadig's study is of interest for both researchers as well as clinicians dealing with West syndrome. It is salutary for researchers to recognise that interventional studies and trials should focus on the most relevant outcome measure such as complete

Prashant Jauhari

pjauhari0@gmail.com

1 Child Neurology Division, Department of Pediatrics, All India Institute of Medical Sciences, New Delhi 110029, India cessation of spasms as a measure of effectiveness. This will bring uniformity and clarity in identifying treatment options with maximum clinical impact. Learning message for the clinicians managing West syndrome is that they must understand that inherently West syndrome is refractory to antiepileptic drugs. Even to the approved first line agents, the response rate is between 50 and $75 \%$, of which $15-33 \%$ relapse [1]. Literature suggests that topiramate, zonisamide and levetiracetam have almost a similar spasm cessation rate of $17-40 \%$, with topiramate rated slightly better than the other two [3]. Only ketogenic diet has a better efficacy in refractory epileptic spasms with spasm cessation rate of 40-50\% [3]. The relapse rate is evenly high with all treatment modalities. Topiramate is a cheap, easily administrable, broad spectrum anti-epileptic, with multiple mechanisms of action and proven efficacy in many refractory epilepsies. Infact, a quarter of Nadig's cases also responded to topiramate [4]. Therefore, it can still be considered as a promising agent. However, what is important is to identify risk factors leading to relapse and the optimal dose at which a sustained response can be obtained. Currently, the most important modifiable risk factor responsible for poor response in West syndrome is the long treatment lag which is even more pronounced in developing countries [5]. Thus, the primary focus of clinicians should be early identification and prompt initiation of definitive therapy. If relapse occurs, then ketogenic diet (if facility is available) or a sequential trial of topiramate, zonisamide and levetiracetam may be given. Till further large-scale comparative studies are available, clinicians should not refute the usage of topiramate in West syndrome, keeping in mind the limited medical options and the invasive and tedious nature of epilepsy surgeries.

\section{Compliance with Ethical Standards}

Conflict of Interest None.

\section{References}

1. Lux AL, Edwards SW, Hancock E, et al. The United Kingdom infantile spasms study (UKISS) comparing hormone treatment with 
vigabatrin on developmental and epilepsy outcomes to age 14 months: a multicentre randomised trial. Lancet Neurol. 2005;4: $712-7$.

2. Song JM, Hahn J, Kim SH, Chang MJ. Efficacy of treatments for infantile spasms: a systematic review. Clin Neuropharmacol. 2017;40:63-84.

3. Kalra V, Gulati S, Pandey RM, Menon S. West syndrome and other infantile epileptic encephalopathies - Indian hospital experience. Brain Dev. 2001;23:593-602.
4. Nadig PL, Sahu JK, Suthar R, Saini A, Sankhyan N. Topiramate as an adjunct in the management of west syndrome. Indian J Pediatr. 2019. https://doi.org/10.1007/s12098-019-03105-0.

5. Jauhari P. Current knowledge, attitude and practice (KAP) of pediatricians on infantile spasms and the way forward. Indian J Pediatr. $2018 ; 85: 823-4$.

Publisher's Note Springer Nature remains neutral with regard to jurisdictional claims in published maps and institutional affiliations. 